\title{
Utility-Based Scheduling Disciplines for Adaptive Applications Over the Internet
}

\author{
Ronaldo M. Salles, Student Member, IEEE, and Javier A. Barria
}

\begin{abstract}
This letter presents packet scheduling disciplines based on application utility functions and network traffic measurements. The disciplines support different classes of adaptive applications over the Internet, providing differentiation, fairness, and dynamic allocation of network resources. They are composed of a decision procedure, where a fairness criterion based on utility functions is used; and a measurement procedure, which considers the statistics involving packet arrivals and departures. The underlying algorithm is then applied to emulate the Proportional Differentiation Services, and is shown-via simulation-that its results outperform the best alternative algorithms published in the literature.
\end{abstract}

Index Terms-Adaptive applications, fairness criteria, measurement-based procedures, scheduling, utility functions.

\section{INTRODUCTION}

D ESPITE all the evolution the Internet has experienced over the years, the majority of its traffic is still best-effort. Even when real-time multimedia applications need to be transported through the network adaptive schemes are suggested where the source itself should adapt to congestion by changing its transmission rate and encoding schemes. Moreover, some popular audio and video applications (e.g., MP3 audio distribution) are based on a store-and-replay fashion generating long-lived file transfers, which are essentially best-effort and not real-time.

Most of todays diversified applications are attended by a single type of service (same-service-to-all), which usually leads to unfair distribution of resources and lack of differentiation. The work in [1] highlights the need for further research within the best-effort service framework and commented about the widespread misconception that best-effort service model implies simple mechanisms (e.g., FIFO queue in network nodes).

In this letter Utility-Based Scheduling (UBS) disciplines are proposed to support different classes of adaptive applications over the Internet. The disciplines differ from other well-known techniques (e.g., WFQ, WRR) since scheduling decisions are directly controlled by utility function values. In this case the utility functions describe the expected level of performance of each class, and rely on the acquisition of on-line traffic statistics to trigger scheduling decisions. Packets are scheduled ac-

Manuscript received December 12, 2001. The associate editor coordinating the review of this letter and approving it for publication was Prof. D. Petr. The work of R. M. Salles was supported by the CNPq, Brazilian Government, under Grant 200049/99-2.

The authors are with the Department of Electrical and Electronic Engineering, Imperial College of Science, Technology and Medicine, London SW7 2BT, U.K. (e-mail: r.salles@ic.ac.uk; j.barria@ic.ac.uk).

Publisher Item Identifier S 1089-7798(02)05092-5.

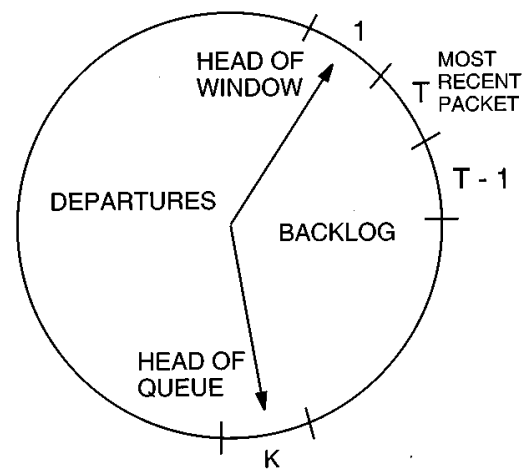

Fig. 1. Measurement window implemented by a circular list.

cording to the utility maximin fairness criterion. This criterion allows network resources to be dynamically allocated while providing differentiation, optimality and fairness among classes of traffic. The scheduling discipline is then used to show how utility functions can be applied to emulate the Proportional Differentiated Services [2]. Finally simulation results show that the proposed discipline outperform other well-known proposals (e.g., [2], [6]).

\section{The MeAsurement Procedure}

The main performance parameter considered in this study is the average packet waiting time (delay). This measure is relevant for any type of application, however, for adaptive applications average performance indices are more important than strict bounds [3].

The use of measures has two major advantages: the ability to handle any input traffic distributions and the capacity to capture input traffic variations even at different time scales. For each class in the system we define a measurement window to store information about most recent arrival/departure statistics. The window is implemented by a circular list with two pointers: head-of-window ( $h w)$ and head-of-queue ( $h q)$. Fig. 1 illustrates the mechanism of the windows.

Both pointers move in the counterclockwise direction as packets enter and leave the system, when the system is empty the pointers are aligned. An incoming packet overwrites the $h w$ register with its new information while the $h q$ packet is the next one to be served. The information stored consists of time-stamps of packets in queue, and waiting times of packets that have already been served. For each window $T_{j}$ of size $\left\|T_{j}\right\|$, packet $i, p_{j}^{i} \in T_{j}$, the following notation is used: $t s_{j}^{i}$ is the packet $i$ time-stamp, $d_{j}^{i}$ is the packet $i$ waiting time, $B_{j}$ is 
the set of backlogged packets in $T_{j}$, and $n_{j}$ is the size of $B_{j}$. The mean delay on window $j$ is given by

$$
\begin{aligned}
D_{j}(t) & =\frac{1}{\left\|T_{j}\right\|} \sum_{i \in T_{j}} d_{j}^{i}=\frac{1}{\left\|T_{j}\right\|}\left(\sum_{i \in B_{j}} d_{j}^{i}+\sum_{i \notin B_{j}} d_{j}^{i}\right) \\
& =\frac{1}{\left\|T_{j}\right\|}\left(n_{j} . t-\sum_{i \in B_{j}} t s_{j}^{i}+\sum_{i \notin B_{j}} d_{j}^{i}\right) \\
& =\frac{1}{\left\|T_{j}\right\|}\left(n_{j} . t-S_{j}+D_{j}^{\mathrm{dep}}\right)
\end{aligned}
$$

where: $j=1, \ldots, C$ classes and $i=1, \ldots,\left\|T_{j}\right\|$. These variables and pointers are updated as indicated below.

$$
\begin{array}{lll}
\frac{\text { At each arrival epoch: }}{n_{j} \leftarrow n_{j}+1} & & \text { At each service epoch: } \\
S_{j} \leftarrow S_{j}+\text { clock } & & S_{j} \leftarrow n_{j}-1 \\
D_{j}^{\text {dep }} \leftarrow D_{j}^{\text {dep }}-d_{j}^{h w} & & d_{j}^{h q} \leftarrow \text { clock }-t s j^{h q} \\
& D_{j}^{\text {dep }} \leftarrow D_{j}^{\text {dep }}+d_{j}^{h q} .
\end{array}
$$

Note that the measure procedure does not need to set or modify any packet field, and that the mean delay as a function of time is directly obtained from (1).

\section{DECISION PROCEDURE-SCHEDULING}

\section{A. Utility Functions}

A formal definition can be given in terms of the set of allocated resources, $A$ (where $a_{i}$ is the amount of resource $i$ ), and the $Q \circ S$ vector, $Q$ (where $q_{i}(A): \Re^{M} \rightarrow \Re$ defines a different $Q \circ S$ measure as a function of those resources)

$$
\begin{array}{ll}
M \text { different resources: } & A=\left\{a_{1}, \ldots, a_{M}\right\} \\
Q \circ S \text { vector: } & Q=\left\{q_{1}, \ldots, q_{N}\right\} \\
\text { Utility function: } & u=f(Q): \Re^{N} \rightarrow[0,1] .
\end{array}
$$

The key idea is that applications do not value the resource by itself but the performance derived from it, therefore, by working directly with the utility functions the resources can be allocated dynamically and in an optimal way. In the reminder of this letter, we consider single variable and monotonically increasing utility function, $Q=\{q\}$ and $u=f(q)$. For adaptive applications it is convenient to define an upper bound to the mean delay and so the $Q o S$ parameter $q$ is expressed as the difference between the maximum allowed delay and the current delay: $q=D^{\max }-D$. For each class $j$, utility values are evaluated using different types of functions: $u_{j}=g_{j}\left(D_{j}^{\max }-D_{j}\right)$, where $D_{j}$ is given by (1).

\section{B. Decision Criterion}

The goal of the scheduling algorithm is to maximize the performance of the system and at the same time guarantee fairness. We use the fairness definition introduced in [4], [7] in the context of data networks and known as maximin criteria. However, instead of using the throughput as the $Q O S$ parameter we are working directly with packet delays. In terms of microeconomic theory, this criterion defines a Social Welfare function that is egalitarian [9]. Then, for $\left\|T_{j}\right\|=T$, the scheduler objective can be characterized by the utility maximin fairness criterion:

$z(t)=\operatorname{maxmin}_{t} g_{j}\left(D_{j}^{\max }-\frac{1}{T}\left(n_{j}(t) \cdot t-S_{j}(t)+D_{j}^{\mathrm{dep}}(t)\right)\right)$.

Even though (2) is defined for all time $t$, scheduler decisions are restricted to departure epochs only, which limits the control actions to those time instants. The optimal solution to (2) can only be obtained if all the events that might happen in the system until the next control epoch are known. Since this is neither possible nor necessary, we propose two different policies for the scheduler: near-optimal and greedy.

Given two consecutive decision epochs, $t$ and $t+\Delta t$, the near-optimal policy aim is to find out how to make a decision on time $t$ that will lead to a solution which is as close as possible to $z(t+\Delta t)$ for a predetermined value of $T$ (e.g., the monitoring timescale [2]). In order to reduce complexity, two basic assumptions are taken: 1) inter-arrival times of packets on each window are i.i.d. and 2) $\Delta t$ is simplified and represents the next arrival in the system or the next service completion time, whichever comes first. At the $i$ th-arrival on queue $j$, the $T$-sized sample mean and variance ${ }^{1}$ of the packet inter-arrival times is computed using the information stored in the measurement windows

$$
\begin{aligned}
\hat{\mu}_{j}^{(i)}= & \left(\operatorname{clock}-t s_{j}^{h w}\right) / T \\
\hat{\sigma}_{j}^{2,(i)}= & \hat{\sigma}_{j}^{2,(i-1)}+\frac{\left(T \cdot \hat{\mu}_{j}^{(i-1)}-\Delta t s_{j}^{\text {old }}\right) \cdot\left(\Delta t s_{j}^{\text {old }}-\Delta t s_{j}^{\text {new }}\right)}{0.5 T(T-1)} \\
& -\frac{\left(\Delta t s_{j}^{\text {old }}\right)^{2}-\left(\Delta t s_{j}^{\text {new }}\right)^{2}}{T}
\end{aligned}
$$

where $\Delta t s_{j}^{\text {old }}=t s_{j}^{(h w+1)}-t s^{(h w)}, \Delta t s_{j}^{\text {new }}=\operatorname{clock}-t s^{(h w-1)}$.

If the sample is large enough [8], we can apply the Central Limit Theorem to estimate $\Delta t$. In our implementation, we consider the values of $Q(x)$, the complementary cdf of a $N(0,1)$, stored in a table to speed up calculations. By letting $I_{j}(k)$ be the sum of $k$ consecutive interarrival times on queue $j$, we have for the next arrival

$$
\begin{aligned}
Q\left(\frac{I_{j}(T)-T \cdot \mu_{j}}{\sigma_{j} \cdot \sqrt{T}}\right) & =\bar{p} \\
I_{j}(T) & =T \cdot \hat{\mu}_{j}+Q^{-1}(\bar{p}) \cdot \sqrt{T} \cdot \hat{\sigma}_{j} .
\end{aligned}
$$

We approximate packet size by an exponential distribution with mean $1 / \beta$. Note that other distributions may also be used without modifying the main algorithm, $\Delta t$ is given by

$$
\Delta t=\min \left[\min _{j}\left\{t s_{j}^{h w}+I_{j}(T)-t\right\},-\log (\bar{p}) / \beta\right]^{+} \text {. }
$$

The Near-Optimal Algorithm:

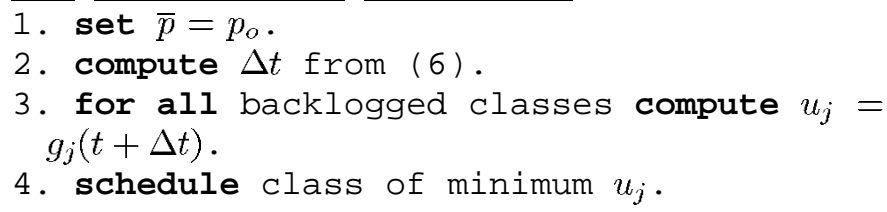

${ }^{1}$ Equation (3) provides a faster update on the sample variance than textbook formulas where all the $T$ samples are taken into account. 
Nr. Sample Windows

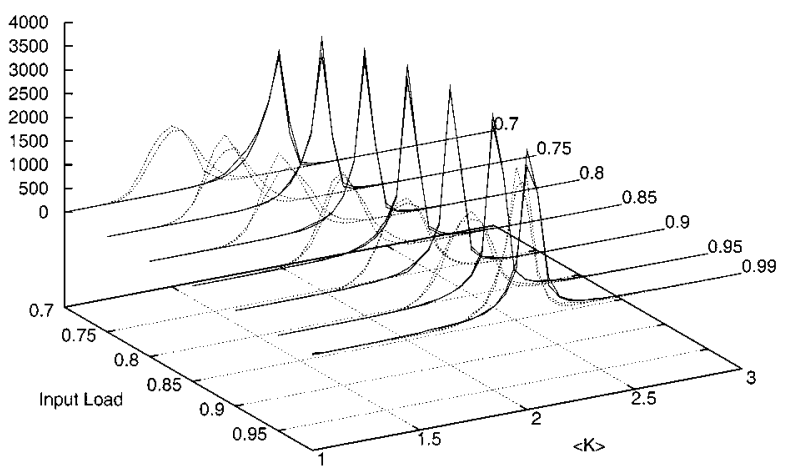

Fig. 2. Balanced traffic, $W M D$ (continuous) and $W T P$ (dashed), $\langle K\rangle=$ $\left\langle K_{12}\right\rangle=\left\langle K_{23}\right\rangle$

The greedy policy is quite simple: at each departure time, select the backlogged queue of lowest utility to serve. This policy is equivalent to set $\Delta t=0$ in the last algorithm.

\section{RESULTS}

In order to define quality spacing among different classes of service, the Proportional Differentiation Services describe the use of multipliers $\left(\delta_{j}\right)$ [2]. Hence, the goal is to perform scheduling in order to maintain the weighted delays of all classes approximately at the same level: $\delta_{1} D_{1} \simeq \delta_{2} D_{2} \simeq \cdots \simeq$ $\delta_{C} D_{C}$. According to [2] one of the most adequate discipline to provide this goal is the Waiting-Time Priority (WTP) which is based on the work of [5]. Packets are scheduled in a high-priority-first basis and priorities increase with time being weighted by a factor $b_{j}: p(t)=b_{j} \cdot\left(t-t_{s}\right)$. The problem with this approach is on finding the optimal weights. The intuitive setting $b_{j}=\delta_{j}$ is only precise when $\rho \rightarrow 1$ (input load near 1 ).

To emulate these services using our framework the class with the lowest multiplier $\left(\delta_{1}\right)$ is taken as reference and the following utility functions are used:

$$
g_{j}=\left(\frac{\delta_{j}}{\delta_{1} \cdot D_{1}^{\max }}\right)\left(D_{j}^{\max }-D_{j}\right) ; \quad D_{j}^{\max }=\frac{\delta_{1} \cdot D_{1}^{\max }}{\delta_{j} .}
$$

Simulation experiments were carried out using Poisson input traffic with loads ranging from 0.7 to 0.99 . A direct comparison to the WTP discipline was carried out using the window mechanism of Section II and the greedy policy. This discipline is named Window Mean Delay (WMD) and constitutes an improvement on our previous work [6].

Three classes of traffic were used: class 1 (lowest priority), class 2 and class 3 (highest priority). The spacing required are defined by the two mean waiting times ratios: $K_{12}=\delta_{2} / \delta_{1}$ and $K_{23}=\delta_{3} / \delta_{2}$. The ratios were set to " 2 " ( $\delta_{1}=1, \delta_{2}=2$ and $\left.\delta_{3}=4\right)$ and sampled from time to time as $\left\langle K_{12}\right\rangle=D_{1} / D_{2}$ and $\left\langle K_{23}\right\rangle=D_{2} / D_{3}$. A good performance is then characterized by small variations around " 2 ." We study two different scenarios: balanced (Fig. 2), where input loads for each class of traffic are identical and, unbalanced (Fig. 3) where the loads differ.

It is clear from the figures that WMD outperformed WTP since it presented the sampled ratio coefficients concentrated closer to the objective value " 2 " and also has a much higher number of samples meeting exactly the objective. WTP presented a poor
Nr. Sample Windows

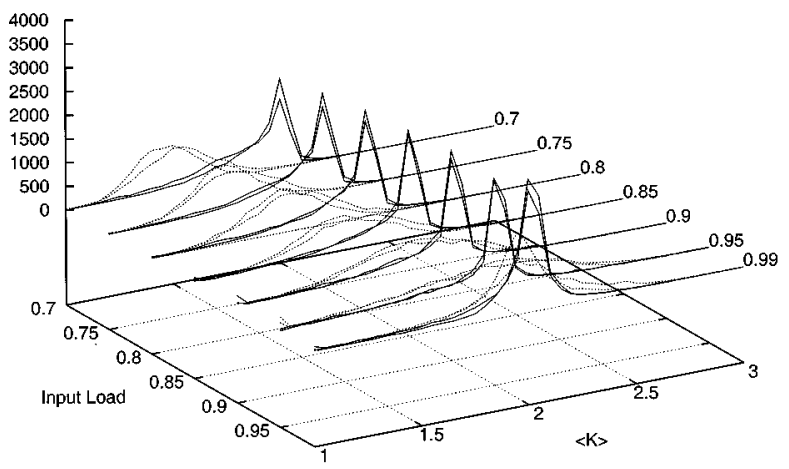

Fig. 3. Unbalanced traffic, $W M D$ (continuous) and $W T P$ (dashed), $\langle K\rangle=$ $\left\langle K_{12}\right\rangle=\left\langle K_{23}\right\rangle$.

performance for the unbalanced case, since the sampled ratio coefficients were spread out along the $\langle K\rangle$ axis. This undesirable behavior was not observed in the WMD plots and hence confirmed the robustness of our algorithm to handle traffic variations without impacting the performance.

\section{CONCLUSIONS}

This work proposes a novel scheduling discipline to provide differentiation and fairness to adaptive applications over the Internet. A Proportional Differentiation Service was implemented and simulation results have shown that the proposed scheme is more effective than currently known algorithms. Our better results can be explained since the WMD scheme generalizes WTP in terms of the parameter $T$ (for $T=1$ and using a greedy policy WMD is reduced to WTP). The utility criterion used-while trying to reach optimality by maximizing the utility functions - also enforces fairness by scheduling the class of minimum utility (2). The effects of that is keeping the utility values approximately at the same level. The freedom to chose other types of utility functions ensures a large degree of flexibility to the proposed algorithm and allows the implementation of other types of services. Finally we note that by controlling the size of the measurement windows it is possible to capture the short-term behavior of the system and provide good performance even for short-lived applications.

\section{REFERENCES}

[1] P. Gevros, J. Crowcroft, P. Kirstein, and S. Bhatti, "Congestion Control Mechanisms and the best-effort service model," IEEE Network Mag., vol. 15 , no. 3 , pp. 16-26, June 2001.

[2] C. Dovrolis and P. Ramanathan, "A Case for relative differentiation services and the proportional differentiation model," IEEE Network Mag., Sep.-Oct. 1999.

[3] H. Zhang, "Service disciplines for guaranteed performance service in packet-switching networks," Proc. IEEE, vol. 83, pp. 1374-1396, Oct. 1995.

[4] D. Bertsekas and R. Gallager, Data Networks, 2nd ed. Englewood Cliffs, NJ: Prentice Hall, 1992.

[5] L. Kleinrock, Queueing Systems-Vol. II. New York: Wiley, 1976.

[6] R. M. Salles and J. A. Barria, "Measurement-based scheduling algorithms for relative service diff.," in IEEE ICT'01, June 2001.

[7] Z. Cao and E. Zegura, "Utility max-min: An application-oriented bandwidht allocation scheme," in IEEE INFOCOM'99, 1999.

[8] A. Papoulis, Probability and Statistics. Englewood Cliffs, NJ: Prentice Hall, 1990

[9] A. Mas-Colell and M. D. Whinston, Microeconomic Theory. New York: Oxford Univ. Press, 1995. 